\title{
A norbornyl route to azasugars: stereoselective synthesis of isofagomine analogues
}

\author{
Goverdhan Mehta* and Narinder Mohal \\ Department of Organic Chemistry, Indian Institute of Science, Bangalore 560 012, India
}

\begin{abstract}
A stereoselective synthesis of new isofagomine analogues has been achieved from a suitably functionalized cyclopentene intermediate extracted from the norbornyl framework. Double reductive amination or inter- and intramolecular $N$-alkylations are the key steps in constructing the piperidine ring. Isofagomine derivatives exhibit moderate inhibitory activity in enzyme assays.
\end{abstract}

Keywords: carbohydrate mimetics; enzyme inhibitors; piperidines; osmylation.

Polyhydroxylated piperidine alkaloids and their synthetic analogues have attracted a great deal of attention in recent years due to their ability to mimic sugars and competitively and selectively inhibit glycosidases and glycosyltransferases, the carbohydrate processing enzymes. ${ }^{1}$ These attributes make hydroxylated piperidines (imino- or azasugars) likely therapeutic agents for the treatment of diseases related to metabolic disorders of carbohydrates such as diabetes, cancer, AIDS and viral infections, where glycoprotein processing is crucial. Typical among the natural products that have shown potent glycosidase inhibition are nojirimycin $\mathbf{1},{ }^{1 \mathrm{a}}$ deoxynojirimycin $2,{ }^{\text {la }}$ homonojirimycin $3^{2 \mathrm{a}}$ and fagomine $4,{ }^{2 \mathrm{~b}}$ representing varying levels of oxygenation and stereochemical patterns on the piperidine ring. The promising profile of hydroxylated piperidines has stimulated a search for newer potent analogues based on this ring system. These efforts have largely focused on restructured monosaccharides with one or more additional side arm(s) and either the anomeric oxygen, ring oxygen, or both, being replaced with nitrogen, which, through protonation, could accommodate positive charge in the transition state to improve inhibition. These efforts have led to the design of glycosylamine $5,{ }^{2 \mathrm{~d}}$ glucosamidine $\mathbf{6},{ }^{\text {lc }}$ isofagomine $7 \mathbf{a}$ and $\mathbf{b}(1-N \text {-iminosugars })^{3}$ and even a 1-azafagomine $\mathbf{8}$, among others, all of which exhibit pronounced glycosidase inhibitory activity. In view of the remarkable inhibition profile of isofagomine $\mathbf{7 a}, \mathbf{b}$ and current interest in its congeners, ${ }^{3}$

\footnotetext{
* Corresponding author. E-mail: gm@orgchem.iisc.ernet.in
} 
we have devised syntheses of homoisofagomine and isofagomine diastereomers and evaluated their activity against glycosidases.
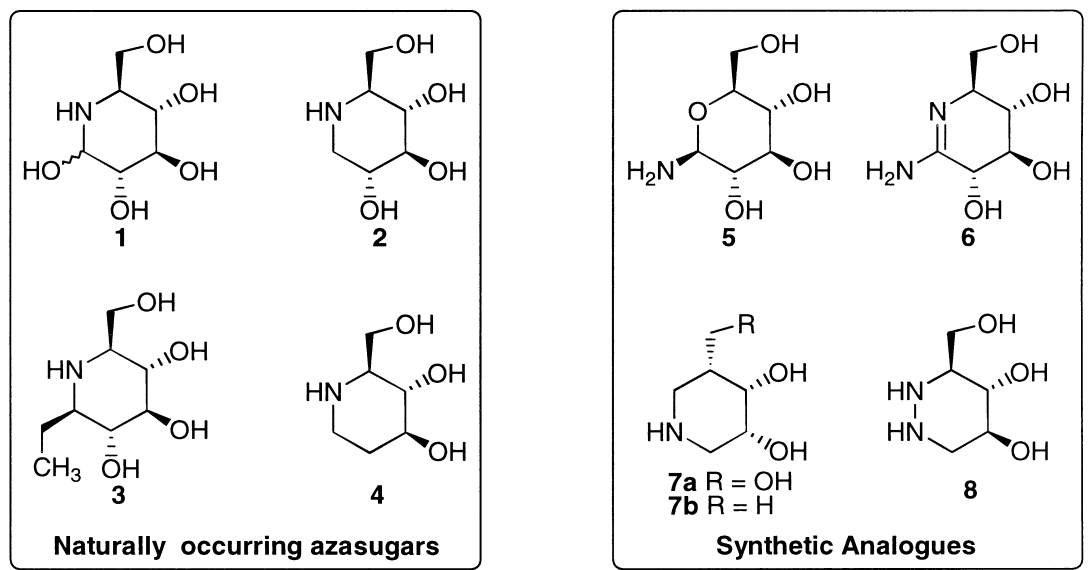

Scheme 1 outlines the retrosynthetic plan for accessing the homoisofagomine 9a and isofagomine 9b analogs from the cyclopentene precursor 11. Oxidative cleavage of the double bond in $\mathbf{1 1}$ was expected to deliver $\mathbf{1 0}$ to set up either double reductive amination or inter- and intramolecular $N$-alkylations to give 9 (Scheme 1). The cyclopentene precursor 11 can in turn be accessed from the fragmentation of the norbornyl derivative $\mathbf{1 2}$ as reported recently by us. ${ }^{4}$

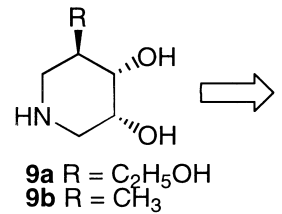

9a $\mathrm{R}=\mathrm{C}_{2} \mathrm{H}_{5} \mathrm{H}$
9b $\mathrm{C} \mathrm{H}_{3}$<smiles>[2H]C(C=O)C1OC(C)(C)OC1C=O</smiles>

10

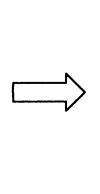

Scheme 1.

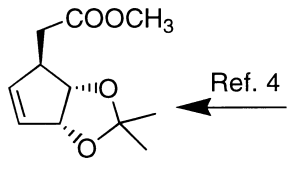

11

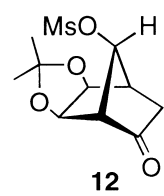

12

LAH reduction and hydroxyl protection transformed 11 to 13 in high yield. The fully protected olefin 13 was subjected to ozonolysis and the intermediate dialdehyde was directly reduced to furnish diol 14. The diol 14 was readily transformed to the dimesylate $\mathbf{1 5}$ to set the stage for interand intramolecular $\mathrm{N}$-alkylations to construct the piperidine ring. Exposure of $\mathbf{1 5}$ to $p$-toluenesulphonamide under phase transfer conditions resulted in smooth cyclization to give $\mathbf{1 6},{ }^{5}$ (Scheme 2). The $N$-tosyl group in $\mathbf{1 6}$ could be removed with sodium naphthalenide and the resulting free amine was characterized as the acetamide $\mathbf{1 7}$, which existed as a mixture of two rotamers. ${ }^{3 e}$ Deprotection manoeuvres in $\mathbf{1 7}$ led to the homoisofagomine $\mathbf{1 9}^{5}$ via the intermediate $\mathbf{1 8}$ (Scheme 2).

To obtain isofagomine, cyclopentene $\mathbf{1 1}$ was elaborated as follows. LAH reduction and acetylation furnished $\mathbf{2 0}$. $\mathrm{OsO}_{4}$ mediated catalytic dihydroxylation of $\mathbf{2 0}$ gave cis-diol 21, essentially as a single diastereomer. Periodate cleavage in $\mathbf{2 1}$ led to an intermediate dialdehyde, which was directly subjected to double-reductive amination ${ }^{6}$ to give piperidine 22 as the major product, Scheme 3. Hydrolysis of acetate 22 and oxidation with TPAP yielded aldehyde 23. Decarbonylation in 23 with the Wilkinson's catalyst was smooth and $\mathbf{2 4}$ was realized as a single diastereomer. Routine deprotection protocols on $\mathbf{2 4}$ delivered isofagomine and its $N$-benzyl derivatives $\mathbf{2 6}^{5}$ and $\mathbf{2 5}{ }^{5}$ respectively (Scheme 3). 


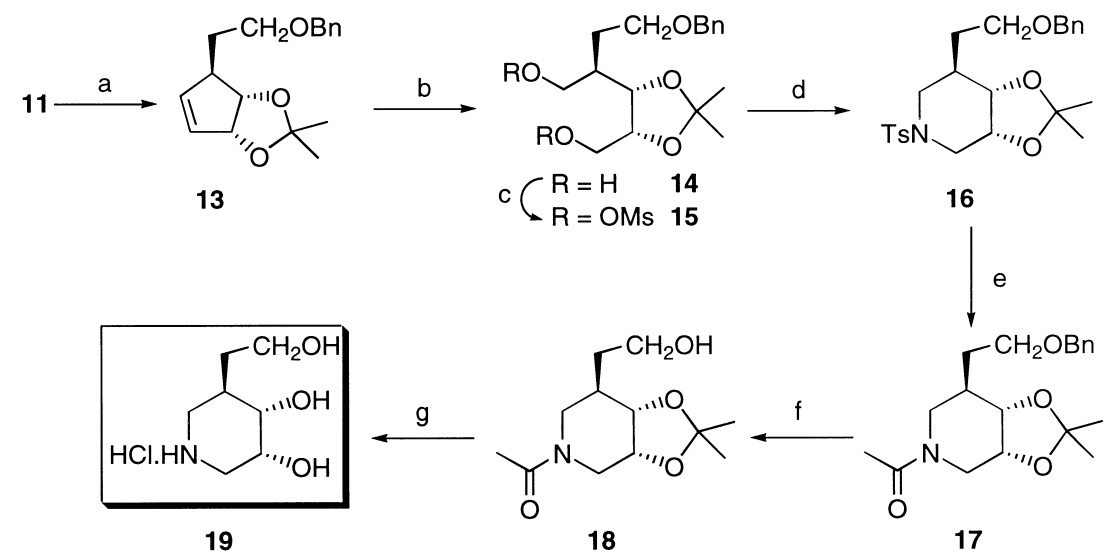

Scheme 2. Reagents and conditions: (a) $\mathrm{LiAlH}_{4}$, THF, $0^{\circ} \mathrm{C}, 30 \mathrm{~min}, 96 \%$; $\mathrm{NaH}, \mathrm{BnBr}, 0^{\circ} \mathrm{C} \rightarrow \mathrm{rt}$, overnight, $\sim 96 \%$; (b) i. $\mathrm{O}_{3}, \mathrm{DCM},-78^{\circ} \mathrm{C}, 5 \mathrm{~min}, \mathrm{DMS}$; ii. $\mathrm{NaBH}_{4}, \mathrm{EtOH}, 6 \mathrm{~h}, 36 \%$ for two steps; (c) $\mathrm{MsCl}, \mathrm{Et}_{3} \mathrm{~N}, \mathrm{DCM},-10^{\circ} \mathrm{C} \rightarrow 0^{\circ} \mathrm{C}$, $>95 \%$; (d) $p$ - $\mathrm{TsNH}_{2},{ }^{n} \mathrm{Bu}_{4} \mathrm{~N}^{+} \mathrm{I}^{-}, \mathrm{KOH}$, benzene: $\mathrm{H}_{2} \mathrm{O}(20: 1), 20 \mathrm{~h}, 61 \%$; (e) sodium naphthalenide, $-78^{\circ} \mathrm{C}, 1 \mathrm{~h} ; \mathrm{Ac}_{2} \mathrm{O}$, py, $90 \%$, (f) $\mathrm{H}_{2}, \mathrm{Pd} / \mathrm{C}\left(10 \%\right.$ ), EtOH, 18 h, 78\%; (g) Et ${ }_{2} \mathrm{O}: \mathrm{HCl}(3: 2), 70^{\circ} \mathrm{C}, 14$ h, $93 \%$

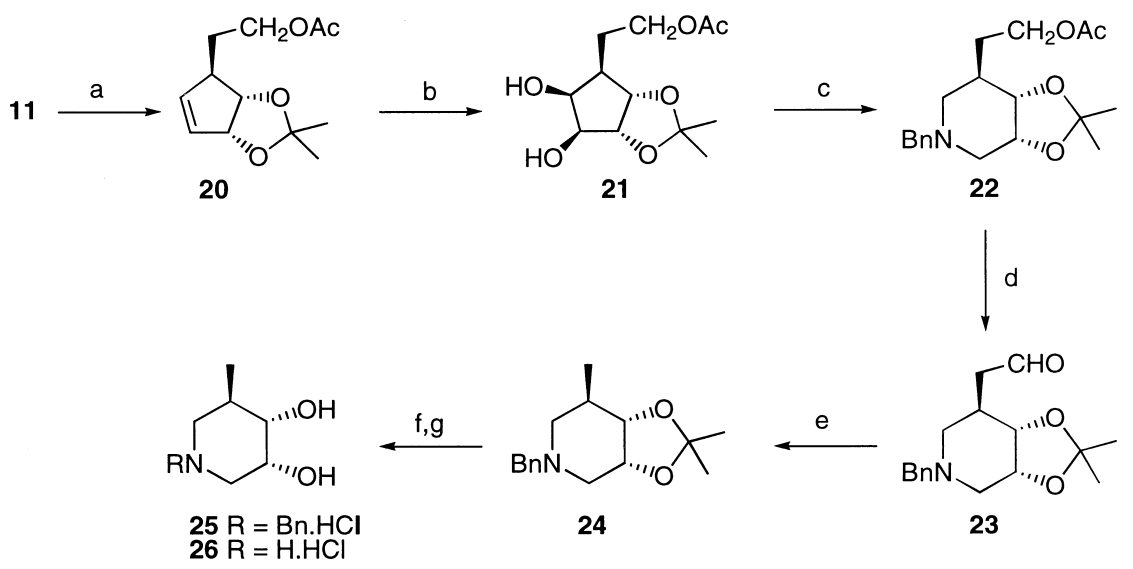

Scheme 3. Reagents and conditions: (a) $\mathrm{LiAlH}_{4}$, THF, $0^{\circ} \mathrm{C}, 30 \mathrm{~min}, 96 \%$; $\mathrm{Ac}_{2} \mathrm{O}, \mathrm{DMAP}, \mathrm{DCM}, 0{ }^{\circ} \mathrm{C}, 45 \mathrm{~min}, \sim 90 \%$; (b) $\mathrm{OsO}_{4}\left(1 \mathrm{~mol} \%\right.$ ), NMMO (50\% aq. sol.), $\mathrm{Me}_{2} \mathrm{CO}: \mathrm{H}_{2} \mathrm{O}, 14 \mathrm{~h}, 84 \%$; (c) i. $\mathrm{NaIO}_{4}\left(1.3\right.$ equiv.), DCM, $0^{\circ} \mathrm{C}, 2$ h, ii. $\mathrm{BnNH}_{2}, \mathrm{AcOH}, \mathrm{NaCNBH}_{3}, \mathrm{MeOH},-10^{\circ} \mathrm{C} \rightarrow \mathrm{rt}, 20 \mathrm{~h}, 49 \%$ for two steps; (d). i. $\mathrm{KOH}, \mathrm{MeOH}, 3 \mathrm{~h}, 92 \%$, ii. ${ }^{n} \mathrm{Pr}_{4} \mathrm{NRuO}_{4}$, NMMO (97\%), mol. sieves $4 \AA$, 2 h, 78\%; (e) $\mathrm{Rh}\left(\mathrm{PPh}_{3}\right)_{3} \mathrm{Cl}$, toluene, reflux, $12 \mathrm{~h}, 60 \%$; (f) $2.5 \% \mathrm{HCl}: \mathrm{Et}{ }_{2} \mathrm{O}$ (1:1), $18 \mathrm{~h},>90 \%$ for 25; (g) $\mathrm{H}_{2}, \mathrm{Pd} / \mathrm{C}(10 \%), \mathrm{EtOH}, 18 \mathrm{~h}, 40 \% ; 2.5 \% \mathrm{HCl} \mathrm{Et}_{2} \mathrm{O}(1: 1), 95 \%$ for 26

New isofagomine analogues 19, 25, 26 were assayed for glycosidase inhibition (Table 1). All measurements were carried out with the corresponding nitrophenyl glycoside substrates in aqueous buffer at the appropriate $\mathrm{pH}$. It was surprising to find that homoisofagomine 19 did not inhibit any of the glycosidases. However, 25 was found to be a moderate to strong inhibitor of all the glycosidases used. Its inhibition of $\alpha$-glucosidase $\left(\mathrm{K}_{\mathrm{i}}=60 \mu \mathrm{M}\right)$ was stronger than of $\beta$-glucosidase $\left(\mathrm{K}_{\mathrm{i}}=1700 \mu \mathrm{M}\right)$. The selectivity in inhibition among $\alpha$-versus $\beta$-galactosidases was much poorer. The debenzylated compound $\mathbf{2 6}$ was found to be a moderate but selective inhibitor of $\beta$-glucosidase. This reversal in selectivities of $\mathbf{2 5}$ and $\mathbf{2 6}$ for $\alpha$ - and $\beta$-glucosidase, respectively, is notable and similar observations have been reported recently for other azasugars. ${ }^{7}$ It is also to be noted that isofagomines $\mathbf{7} \mathbf{b}^{3 \mathrm{~d}}$ and $\mathbf{2 6}$ exhibit very similar $\beta$-glucosidase inhibition (see Table 1), 
Table 1

Inhibition constants ${ }^{\mathrm{a}, \mathrm{b}}\left(\mathrm{K}_{\mathrm{i}}\right)$ in $\mu \mathrm{M}$

\begin{tabular}{|c|c|c|c|c|}
\hline \multirow[b]{2}{*}{ Enzyme } & \multicolumn{4}{|c|}{ compound } \\
\hline & 19 & 25 & 26 & $7 b$ \\
\hline $\begin{array}{l}\alpha \text {-glucosidase } \\
\text { (yeast) }\end{array}$ & $\mathrm{NI}$ & 60 & $\mathrm{NI}$ & $\mathrm{NI}$ \\
\hline $\begin{array}{l}\beta \text {-glucosidase } \\
\text { (sweet almond) }\end{array}$ & $\mathrm{NI}$ & 1700 & 140 & 120 \\
\hline $\begin{array}{l}\alpha \text {-galactosidase } \\
\text { (green coffee beans) }\end{array}$ & $\mathrm{NI}$ & 89 & $\mathrm{NI}$ & - \\
\hline $\begin{array}{l}\beta \text {-galactosidase } \\
(E . \text { Coll })\end{array}$ & $\mathrm{NI}$ & 180 & $\mathrm{NI}$ & $\mathrm{NI}$ \\
\hline
\end{tabular}

${ }^{\mathrm{a}}$ Each $200 \mu \mathrm{L}$ assay contained indicated enzyme, inhibitor in water(2-3 $\mathrm{mM})$ and nitrophenyl glycosides $(2-2.5 \mathrm{mM})$ in appropriate buffer at optimal temp and $\mathrm{pH}$ of each enzyme. ${ }^{\mathrm{b}}$ Inhibiton constants were determined using Dixon plots of inhibition data. ${ }^{\mathrm{c}}$ No inhibition is observed up to $1 \mathrm{mM}$ conc. of inhibitor.

indicating that stereochemical disposition of substituents in isofagomine series has little modulating effect on $\beta$-glucosidase inhibition. ${ }^{3 \mathrm{~d}}$

In conclusion, we have amplified the synthetic utility of the cyclopentanoid building block 11 by devising stereoselective routes to isofagomine analogues. Our preliminary results of enzymatic assays reveal the importance of $N$-substitution in modulating selectivity and inhibition efficacy in azasugars.

\section{Acknowledgements}

We thank Dr Utpal Tatu, Department of Biochemistry, IISc, Bangalore, for useful discussions and valuable suggestions. N.M. thanks CSIR for the award of a Research Fellowship.

\section{References}

1. Recent Reviews: (a) Hughes, A. B.; Rudge, A. J. Nat. Prod. Rep. 1994, 135. (b) Jacob, G. S. Curr. Opin. Struct. Biol. 1995, 605. (c) Ganem, B. Acc. Chem. Res. 1996, 29, 340. (d) Bols, M. Acc. Chem. Res. 1998, 31, 1. (e) Heightman, T. D.; Vasella, A. T. Angew. Chem., Int. Ed. 1999, 38, 750. (f) Sears, P.; Wong, C.-H. ibid. 1999, 38, 2300 .

2. (a) Ikeda, K.; Takahashi, M.; Nishida, M.; Miwa, M.; Kizu, H.; Kameda, Y.; Arisawa, M.; Watson, A. A.; Nash, R. J.; Fleet, G. W. J.; Asano, N. Carbohydr. Res. 2000, 323, 73. (b) Asano, N.; Tomioka, E.; Kizu, H.; Matsui, K. ibid. 1994, 253, 235. (c) Kato, A.; Asano, A.; Kizu, H.; Matsui, K. J. Nat. Prod. 1997, 60, 312. (d) Legler, G. Adv. Carbohydr. Chem. Biochem. 1990, 48, 319

3. (a) Ichikawa, Y.; Igarashi, Y., Tetrahedron Lett. 1995, 36, 4585. (b) Ichikawa, Y.; Igarashi, Y.; Ichikawa, M.; Suhara, Y. J. Am. Chem. Soc. 1998, 120, 3007. (c) Jespersen, T. M.; Dong, W.; Sierks, M. R.; Skrydsurp, T.; Lundt, I.; Bols, M. Angew. Chem., Int. Ed. Engl. 1994, 33, 1778. (d) Hansen, A.; Tagmose, T. M.; Bols, M. Chem. Commun. 1996, 2649. (e) Ernholt, B. V.; Thomson, I. B.; Jensen, K.; Bols, M. Synlett 1999, 6, 701. (f) Hensen, S. U.; Bols, M. J. Chem. Soc., Perkin Trans. 1 2000, 911 and references cited therein. 
4. Mehta, G.; Mohal, N. Tetrahedron Lett. 1999, 40, 5791.

5. All new compounds reported here were racemic and were characterized on the basis of their spectral data $\left({ }^{1} \mathrm{H}\right.$ and ${ }^{13} \mathrm{C}$ NMR, IR, Mass). Selected spectral data $\left({ }^{1} \mathrm{H}\right.$ NMR, ${ }^{13} \mathrm{C}$ NMR $): 16: \delta_{\mathrm{H}}\left(300 \mathrm{MHz}, \mathrm{CDCl}_{3}\right): 7.62(2 \mathrm{H}, \mathrm{d}, J=8.1$ $\mathrm{Hz}), 7.36-7.31(5 \mathrm{H}, \mathrm{m}, \mathrm{Ar}-\mathrm{H}), 7.28(2 \mathrm{H}, \mathrm{d}, J=8.1 \mathrm{~Hz}), 4.5(2 \mathrm{H}, \mathrm{ABq}, J=12 \mathrm{~Hz}), 4.18(1 \mathrm{H}, \mathrm{dd}, J=5.7,6.0 \mathrm{~Hz})$, $3.85(1 \mathrm{H}, \mathrm{dd}$ as t, $J=5.1 \mathrm{~Hz}), 3.64-3.55(2 \mathrm{H}, \mathrm{m}), 3.41(1 \mathrm{H}, \mathrm{dd}, J=5.4,12 \mathrm{~Hz}), 3.08(1 \mathrm{H}, \mathrm{dd}, J=3.6,12.0 \mathrm{~Hz}), 2.98$ $(1 \mathrm{H}, \mathrm{dd}, J=6.3,12.3 \mathrm{~Hz}), 2.88(1 \mathrm{H}, \mathrm{dd}, J=6.3,12.3 \mathrm{~Hz}), 2.41(3 \mathrm{H}, \mathrm{s}), 2.19-2.10(1 \mathrm{H}, \mathrm{m}), 1.83-1.62(2 \mathrm{H}$, series of m), $1.31(3 \mathrm{H}, \mathrm{s}), 1.29(3 \mathrm{H}, \mathrm{s}) ; \delta_{\mathrm{C}}\left(75 \mathrm{MHz}, \mathrm{CDCl}_{3}\right): 143.47(\mathrm{C}), 138.35(\mathrm{C}), 133.94(\mathrm{C}), 129.62(\mathrm{CH}, 2 \mathrm{C}), 128.37$ $(\mathrm{CH}, 2 \mathrm{C}), 127.62(\mathrm{CH}, 2 \mathrm{C}), 127.56(\mathrm{CH}), 127.52(\mathrm{CH}, 2 \mathrm{C}), 108.87(\mathrm{C}), 76.54(\mathrm{CH}), 72.94\left(\mathrm{CH}_{2}\right), 70.79(\mathrm{CH}), 63.58$ $\left(\mathrm{CH}_{3}\right), 47.76\left(\mathrm{CH}_{2}\right), 45.80\left(\mathrm{CH}_{2}\right), 34.46(\mathrm{CH}), 30.48\left(\mathrm{CH}_{2}\right), 28.02\left(\mathrm{CH}_{3}\right), 26.04\left(\mathrm{CH}_{3}\right), 21.48\left(\mathrm{CH}_{3}\right) .19: \delta_{\mathrm{H}}(300$ $\left.\mathrm{MHz}, \mathrm{D}_{2} \mathrm{O}\right)$ : $4.01(1 \mathrm{H}$, br. s), 3.56-3.44 $(3 \mathrm{H}$, series of $\mathrm{m}), 3.34-3.25(2 \mathrm{H}$, series of $\mathrm{m}), 3.04(1 \mathrm{H}, \mathrm{d}, J=13.5 \mathrm{~Hz})$, $2.68(1 \mathrm{H}, \mathrm{dd}$ as t, $J=13 \mathrm{~Hz}), 2.09-1.99(1 \mathrm{H}, \mathrm{m}), 1.88-1.76(1 \mathrm{H}, \mathrm{m}), 1.40-1.28(1 \mathrm{H}, \mathrm{m}) ; \delta_{\mathrm{C}}\left(75 \mathrm{MHz}, \mathrm{D}_{2} \mathrm{O}\right): 71.68$ $(\mathrm{CH}), 66.12(\mathrm{CH}), 59.93(\mathrm{CH}), 48.80\left(\mathrm{CH}_{2}\right), 47.13\left(\mathrm{CH}_{2}\right), 32.81(\mathrm{CH}), 31.98\left(\mathrm{CH}_{2}\right) .25: \delta_{\mathrm{H}}\left(300 \mathrm{MHz}, \mathrm{D}_{2} \mathrm{O}\right): 7.38$ $(5 \mathrm{H}, \mathrm{m}), 4.24\left(1 \mathrm{H}, \frac{1}{2} \mathrm{ABq}, J=13.3 \mathrm{~Hz}\right), 4.15\left(1 \mathrm{H}, \frac{1}{2} \mathrm{ABq}, J=13.3 \mathrm{~Hz}\right), 3.97(1 \mathrm{H}$, br. s), 3.32-3.28 $(3 \mathrm{H}, \mathrm{m}), 3.01(1 \mathrm{H}$, d, $J=13 \mathrm{~Hz}), 2.67(1 \mathrm{H}$, dd as t, $J=13 \mathrm{~Hz}), 2.13-2.07(1 \mathrm{H}, \mathrm{m}), 0.86(3 \mathrm{H}, \mathrm{d}, J=6.6 \mathrm{~Hz}) ; \delta_{\mathrm{C}}\left(75 \mathrm{MHz}, \mathrm{D}_{2} \mathrm{O}\right): 132.05$ $(\mathrm{CH}, 2 \mathrm{C}), 130.96(\mathrm{CH}), 129.96(\mathrm{CH}, 2 \mathrm{C}), 128.90(\mathrm{C}), 73.10(\mathrm{CH}), 66.58(\mathrm{CH}), 60.79\left(\mathrm{CH}_{2}\right), 57.04\left(\mathrm{CH}_{2}\right), 56.38$ $\left(\mathrm{CH}_{2}\right), 30.53(\mathrm{CH}), 14.49\left(\mathrm{CH}_{3}\right) .26: \delta_{\mathrm{H}}\left(300 \mathrm{MHz}, \mathrm{D}_{2} \mathrm{O}\right): 3.99(1 \mathrm{H}$, br. s), $3.33(1 \mathrm{H}, \mathrm{dd}, J=2.7,10.5 \mathrm{~Hz}), 3.29-3.15$ $(2 \mathrm{H}$, series of m), $3.02(1 \mathrm{H}, \mathrm{d}, J=13 \mathrm{~Hz}), 2.59(1 \mathrm{H}, \mathrm{dd}$ as t, $J=12.6 \mathrm{~Hz}), 2.10-2.0(1 \mathrm{H}, \mathrm{m}), 0.88(3 \mathrm{H}, \mathrm{d}, J=6.6 \mathrm{~Hz})$; $\delta_{\mathrm{C}}\left(75 \mathrm{MHz}, \mathrm{D}_{2} \mathrm{O}\right): 73.40(\mathrm{CH}), 66.16(\mathrm{CH}), 49.11\left(\mathrm{CH}_{2}\right), 48.80\left(\mathrm{CH}_{2}\right), 30.26(\mathrm{CH}), 14.60\left(\mathrm{CH}_{3}\right)$.

6. Baxter, E. W.; Reitz, A. B. J. Org. Chem. 1994, 59, 3175.

7. Butters, T. D.; van den Broek, L. A. G. M.; Fleet, G. W. J.; Krulle, T. M.; Wormald, M. R.; Dwek, R. A.; Platt, F. M. Tetrahedron: Asymmetry 2000, 11, 113. 